\title{
La noción de tópico en la evolución de la duplicación clítica
}

\author{
Margot Vivanco \\ Universidad Complutense de Madrid \\ margotvivanco@filol.ucm.es
}

\section{RESUMEN}

El fenómeno de la duplicación clítica está estrechamente ligado a la noción de 'tópico'. En este trabajo dicha noción será empleada como herramienta fundamental para analizar, a través del corpus, el proceso de gramaticalización del pronombre átono como afijo de concordancia. Asimismo, los datos evidenciarán que esta noción opera de manera determinante no sólo en el ámbito del complemento duplicado, sino en el de toda la construcción, de tal manera que el bajo grado de topicidad del sujeto será aducido como uno de los factores desfavorecedores de la duplicación clítica en su evolución.

Palabras clave: Duplicación clítica, tópico, concordancia, gramaticalización, sujeto.

\begin{abstract}
Clitic doubling phenomena are closely related to the notion of 'topic'. Thus, this notion is going to be the tool used in this paper in order to analize, across the corpus, the grammaticalization process turning the clitic into an agreement affix. Moreover, the data will show that topicality is not only a key notion dealing with the doubled object itself, but with the whole sentence, so that the subject's low degree of topicality will be argued as one of the non helping factors for clitic doubling during its evolution.
\end{abstract}

Key words: Clitic doubling, topic, agreement, grammaticalization, subject.

\section{INTRODUCCIÓN}

Uno de los resultados de la poligramaticalización del determinante latino ILLE es el pronombre átono le (lo < ILLUM, la < ILLA). En este proceso, se han dado ya pérdidas de cuerpo fónico, de contenido semántico (paso del significado deíctico al anafórico) y de libertad posicional (clítico); pero la duplicación clítica supone un paso más allá, haciendo de un elemento gramatical (el pronombre átono) un elemento aún más gramatical (afijo de concordancia).

En español moderno, la duplicación clítica es obligatoria con los objetos (directos e indirectos) topicalizados, así como con los pronominales; la ausencia de duplicación en esos casos resulta agramatical (1.a-d). 
(1) a. A Juan *(1o) vi.

b. A María *(le) di el regalo.

c. *(Lo) vi a él.

d. *(Le) di el regalo a ella.

(2) Le di el regalo a \{María / mi hermana\}

Por otra parte, la duplicación del OI está haciéndose cada vez más frecuente (2), lo cual ha sido analizado en numerosas ocasiones ${ }^{1}$ como un paso hacia el desarrollo de una conjugación objetiva en español. No obstante, tal y como se acaba de señalar, la duplicación es obligatoria con los dos tipos de objeto -no sólo con el indirectosiempre y cuando estos tengan un alto grado de topicidad -dislocación a la izquierda, pronombres personales-, lo cual hace pensar que la concordancia se está estableciendo con el tópico (Girón Alconchel: 2002), no con la función de objeto indirecto per se. Desde una perspectiva diacrónica, es sabido que la duplicación del OI se extiende de acuerdo con las siguientes escalas de topicidad (Givón: 1976):

TABLA 1

ESCALAS DE TOPICIDAD

Nominativo $>$ Dativo $>$ Acusativo

Agente $>$ Experimentante $>$ Objeto

Pronombre personal $>$ Nombre propio $>$ Nombre común

Tema / Comentario

Animado > Inanimado

Definido $>$ Indefinido

Específico $>$ Inespecífico

La duplicación es uno de los muchos mecanismos de la lengua vinculados a los participantes del discurso, y a su relevancia dentro de éste: los referentes humanos, conocidos, especificados, tienen prioridad a la hora de convertirse en tópicos, y la topicidad es quizá la noción pragmática con mayor repercusión o codificación sintáctica.

Otros fenómenos subordinados a estas escalas, y operantes en el mismo ámbito funcional -el del objeto-, son el leísmo y complemento directo preposicional (CDP), que desdibujan la diferenciación formal del OD y OI. Ambos procesos toman las marcas propias del OI (preposición $a$, pronombre le) para establecer un marcado diferencial del OD humano, dando prioridad a los rasgos de animacidad y definitud sobre la distinción funcional. La idea de que la proliferación de estos fenómenos alienta la extensión de la duplicación del OI como mecanismo para volver a

\footnotetext{
${ }^{1}$ Véanse A. Llorente y J. Modéjar (1974), y A. Enrique Arias (2002) entre otros.
} 
diferenciarlo del OD está comúnmente aceptada en la bibliografía ${ }^{2}$; sin embargo, ésta no puede considerarse como la única causa determinante ${ }^{3}$, pues la duplicación es asimismo, como el CDP y el leísmo, una marca de topicidad, razón por la cual se da también con el OD, aunque de manera mucho más restringida ${ }^{4}$.

Además de su obligatoriedad en los contextos citados, hay otra razón fundamental para analizar la duplicación como un primer paso hacia la concordancia. La función original del clítico es reemplazar a un complemento ausente, no coaparecer con él ${ }^{5}$; para autores como Weissenrieder (1995) o GarcíaMiguel (1991), entender la duplicación como concordancia responde de manera natural la pregunta acerca de cuál es el elemento redundante en la construcción, pues la morfología de concordancia es la que se convierte en obligatoria, y la realización del argumento es lo prescindible.

Atendiendo por un momento a la concordancia subjetiva, es sabido que el español, en tanto que lengua pro-drop, expresa sus sujetos como SSNN plenos sólo cuando éstos contienen información nueva $\mathrm{o}$ aparecen en un contexto pragmáticamente marcado -enfático, etc.-. Los ejemplos como el de (2) funcionan de la misma manera, lo cual lleva a pensar, de acuerdo con Weissenrieder (1995) y otros, que la duplicación clítica es un mecanismo análogo a la concordancia subjetiva, y que supone por tanto la creación de un afijo capaz de expresar las funciones sintácticas en el verbo ${ }^{6}$.

\footnotetext{
${ }^{2}$ Véase por ejemplo M. Flores y C. Melis (2006).

${ }^{3}$ Otra posible causa argumentada en la bibliografía es el uso del clítico para marcar la inclusión de un dativo no argumental en la estructura oracional. En esta línea véanse S. Ibáñez Cerdá (2008) y F. Barrio de la Rosa (2008).

${ }^{4} \mathrm{La}$ diferenciación de las funciones juega también un papel importante en la extensión asimétrica de la duplicación en el OD y el OI.

${ }^{5}$ Véanse L. Tesinière (1959), A. Llorente y J. Modéjar (1974), M. Suñer (1988) entre otros.

${ }^{6}$ En la misma línea, S. Becerra Bascuñán (2006) observa que la duplicación clítica manifiesta una adaptación a la tipología lingüística (pro-drop); es decir, la duplicación, como todo cambio, se produce en relación a unas necesidades comunicativas, pero siempre de acuerdo con las propiedades del sistema.
} 
TABLA 2 (Weissenrieder: 1995)

CONCORDANCIA SUBJETIVA - DUPLICACIÓN CLÍTICA

\begin{tabular}{|l|l|c|}
\hline $\mathrm{V}_{\text {morf }}+\mathrm{SN}$ & Sujeto + Concordancia & Clítico + Concordancia \\
\hline $\mathrm{V}_{\text {morf }}+$ pron. & Hablaron Juan y Pepe & $\begin{array}{c}\text { Les hablaron a Juan y a } \\
\text { Pepe }\end{array}$ \\
\hline $\mathrm{V}_{\text {morf }}+\varnothing$ & Hablaron ellos & Les hablaron a ellos \\
\hline & Hablaron & Les hablaron $\varnothing$ \\
\hline
\end{tabular}

En la sección 2 se analizará a través del corpus ${ }^{7}$ cómo se produce diacrónicamente la consolidación de la duplicación clítica como concordancia.

En la sección 3, se analizarán por otra parte aquellos casos en los que la duplicación es esperable, y sin embargo no se produce; para ello se atenderá al contexto sintáctico-discursivo, y se subrayará la importancia de las propiedades del sujeto oracional -bajo grado de topicidad- en relación con la ausencia de duplicación en el objeto.

\section{LA DUPLICACIÓN CLÍTICA COMO CONCORDANCIA}

La duplicación clítica atraviesa diferentes estadios hasta consolidarse plenamente como concordancia. Así, comienza por subrayar la prominencia de un elemento en relación con el grado de topicidad y con contextos pragmáticamente marcados (Girón Alconchel: 2002); en el corpus, este estadio está representado por Torres Villarroel y Mesonero Romanos (ss. XVIII y XIX). A medida que se hace más frecuente, es decir, regular, la construcción pierde carga pragmática, de manera que hay un momento en el que el énfasis pragmático deja de ser un factor influyente sobre la duplicación. Es entonces cuando el hecho de que el clítico y el SN pleno coaparecezcan deja de resultar redundante y se vuelve regular. Dentro del corpus, podrá observarse este momento de inflexión a través de los ejemplos tomados de Pío Baroja. En un estadio más avanzado -representado en el corpus por Javier Marías-, es el SN pleno lo que resulta redundante, de manera que tiende a ser omitido a menos que contenga información nueva. Éste es el momento en el que la concordancia está verdaderamente fijada.

\footnotetext{
${ }^{7}$ Las obras tomadas como corpus para este estudio son: Vida (1743), de Torres Villarroel; Escenas Matritenses (1851), de Mesonero Romanos; Las inquietudes de Shanti Andía (1911), de Pío Baroja; y Negra espalda del tiempo (1998), de Javier Marías. Todas ellas pertenecen a autores peninsulares, y juntas abarcan los tres últimos siglos de la historia del español; si bien la duplicación clítica es un fenómeno que se atestigua desde la Edad Media, éste es el periodo fundamental para su expansión.
} 
Así es como la duplicación clítica se gramaticaliza como concordancia con los pronombres personales -alto grado de topicidad: animados, definidos y específicosjunto con la dislocación a la izquierda, éste es el primer contexto en el que la duplicación es obligatoria. En un primer momento, la duplicación está favorecida por diferentes factores, y se relaciona con contenidos pragmáticos -contraste, énfasis, afectación-. Entonces se vuelve regular y no marcada, y finalmente se consolida de manera que el pronombre personal resulta redundante:

(3) Le di a él [marcado] > le di a él [no marcado] > le di [no marcado] / le di a él [marcado]

(4) Duplicación favorecida por ciertos contextos > duplicación regular / concordancia $>$ concordancia plena (la morfología basta para referir al complemento léxico)

El siguiente paso es la extensión de la duplicación a SSNN animados, definidos, específicos y singulares. Hoy, la duplicación con este tipo de complementos es regular, pero el SN no resulta redundante aún. Mediante el análisis del corpus se podrá observar lo dicho hasta aquí, y se apreciará asimismo cómo la extensión de la duplicación clítica se consolida en un contexto -el de los complementos altamente topicales- antes de haber comenzado a operar de manera significativa sobre complementos con un bajo grado de topicidad, es decir, inanimados, indefinidos, inespecíficos, etc.

\subsection{La extensión de la duplicación clítica en el OI}

En la obra de Torres Villarroel (TV), la duplicación (DCL) del OI no está generalizada, y supone un $30 \%$ respecto del total de OOII (no topicalizados) estudiados. La no duplicación (70\%) es mayoritaria tanto entre los OOII animados como entre los inanimados; y de hecho, el único caso de duplicación de un OI inanimado puede considerarse como una personificación:

(5) [...] que es cuanto se le puede pedir a una edad en la que sola la memoria tiene más discernimiento [...] $(\mathrm{TV}, 92)$

TABLA 3

DCL DE OOII ANIMADOS / INANIMADOS EN TORRES VILLARROEL

\begin{tabular}{|l|l|l|}
\hline & DCL & No DCL \\
\hline OOII Animados 21 (27) & $7(21) \mathbf{3 3 \%}$ & $14(21) \mathbf{6 7 \%}$ \\
\hline OOII Inanimados 6(27) & $1(6) \mathbf{1 7 \%}$ & $5(6) \mathbf{8 3} \%$ \\
\hline
\end{tabular}


Centrando la atención en los OOII animados, se observa que la duplicación preferida es la de los pronombres personales (6) -no aparece ningún ejemplo de no duplicación en este contexto-, y la de los sintagmas definidos, específicos, singulares, si bien se sigue tendiendo más a la no duplicación incluso en estos contextos (7.a-b). Dos de los tres casos de duplicación de OOII con pronombre tónico son claramente enfáticos, como se observa en el ejemplo (6), lo cual es un dato a favor de las ideas anteriormente expuestas.

(6) Los jácaros de capotillo y guadejeño, y el suizo con los bigototes, el sable y las pistolas, son hombres con miedo; y el que justamente presumo en ellos me quita a mi el que me pudieran persuadir sus apatuscos, sus armas y sus juramentos. (TV, 127)

(7) a. Dediqué las conclusiones al excelentísimo señor príncipe de Chalamar $[\ldots](\mathrm{TV}, 133)$

b. [...] y al punto partí a rogar a mi clérigo contrabandista que me soltase $[\ldots](\mathrm{TV}, 150)$

TABLA 4

DCL OI TORRES VILLARROEL

\begin{tabular}{|l|l|l|}
\hline & DCL OI animado & No DCL OI animado \\
\hline Det. Esp. Sg. Pron. tónico & $3(21) \mathbf{1 3} \mathbf{5 \%}$ & --- \\
\hline Det. Esp. Sg. & $1(21) \mathbf{5 \%}$ & $7(21) \mathbf{3 3 \%}$ \\
\hline Det. Esp. Pl. & $1(21) \mathbf{5 \%}$ & $1(21) \mathbf{5 \%}$ \\
\hline Det. Inesp. Sg. & ----- & $1(21) \mathbf{5 \%}$ \\
\hline Det. Inesp. P1. & ----- & $2(21) \mathbf{1 0} \%$ \\
\hline Indet. Inesp. Sg. & $2(21) \mathbf{1 0} \%$ & ---- \\
\hline Indet. Inesp. Pl. & ---- & $3(21) \mathbf{1 3} \mathbf{5} \%$ \\
\hline
\end{tabular}

Por otra parte, en Mesonero Romanos ${ }^{8}$ (MR) la no duplicación supone un $85 \%$ con respecto al total de OOII animados. La duplicación es obligatoria cuando se trata de un pronombre tónico (8.a) -el 9\% de los OOII animados-, mientras que la

\footnotetext{
${ }^{8}$ El estudio de este autor se ha realizado sobre las siguientes Escenas matritenses: "La Calle Toledo" (CT), "La Romería de San Isidro" (RSI), "El Prado" (P), "El Extranjero en su Patria" (EP), "Paseo por las Calles" (PC), "El Romanticismo y los Románticos" (RR), "Las Sillas del Prado" (SP), "El Recién Venido" (RV), y "La Posada, o España en Madrid" (PEM).
} 
duplicación de otros OOII determinados, específicos y singulares (8.b-c) supone el $6 \%$; este último tipo de complementos tiende mayoritariamente $(36 \%$ sobre el total de animados) a no duplicarse (9.a-c), incluidos también los nombres propios (9.a en contraste con 8.c):

(8) a. Por lo demás, eso le daba a él de los tratados de economistas célebres [...] (MR, 136, PEM)

b. - Déselos a ese mi criado [...] (MR, 127, RV)

c. Hablárasle a Apolo de herejes judaizantes o de moriscos recién convertidos [...] (MR, 106, SP)

(9) a. [...] en donde dijo el mozo a Juan que, mientras llamaba a la puerta, esperase [...] (MR, 127, RV)

b. [...] y las niñas declaran al pobre galán que por una consecuencia desgraciada su mamá no puede volver a pie... (MR, 49, RSI)

c. [...] en todas ellas venía a decir a su amante, con la mayor ternura, que era preciso que se muriesen para ser felices $[\ldots](\mathrm{MR}, 90, \mathrm{RR})$

Un $60 \%$ de los casos de duplicación del OI -que, como hemos visto, sólo se produce en sintagmas con un alto grado de topicidad- se encuentra en contextos enfáticos y/o contrastivos (10), además del caso ilustrado en (8.b), que sin ser una verdadera dislocación a la derecha resulta redundante.

(10) ¿Y qué me sirven esas cualidades y ese aplauso, y qué le sirven a él tampoco $[\ldots]$ ? (MR, 66, EP)

Por otro lado, y como cabía esperar, los contextos más reacios a la duplicación tanto en Torres Villarroel como en Mesonero Romanos son aquellos con un bajo grado de topicidad. Así, en Torres Villarroel es difícil hallar duplicación con complementos indeterminados y/o indefinidos, especialmente cuando son, además, plurales (11.a-b). En (12) aparecen coordinados dos ejemplos de duplicación con OOII de este tipo:

(11) a. [...] leí y enseñé dos años a bastante número de discípulos [...] (TV, 133)

b. Al tiempo que expresaba mis rendimientos, escondía mi persona; y las más de las veces, dedicaba a los héroes más elevados, a los ausentes [...] $(\mathrm{TV}, 123)$

(12) [...] para dárselo a quien me quiera hechizar, o regalársele a una bruja $[\ldots](\mathrm{TV}, 126)$ 
La duplicación del OI inanimado es asimismo nula en Mesonero Romanos. Siguen sin duplicarse estos OOII aun estando personificados y siendo determinados, específicos y singulares:

(13)a. [...] pareció dar vida y movimiento a aquel solitario recinto [...] (MR, 99, SP)

b. [...] quiso entregarlas al fuego [sus composiciones] [...] (MR, 95, RR)

De igual modo, y para concluir, los OOII plurales o colectivos (14.a-b), inespecíficos (14.c) y/o indeterminados (14.d) nunca se duplican:

(14)a. [...] y dando al público en mutilados trozos el esqueleto de tan gigantesca composición. (MR, 95, RR)

b. [...] y daba a los madrileños el grato espectáculo de su hermosa faz [...] (MR, 99, SP)

c. En cuanto al drama, no fue posible encontrarle, por haberle prestado mi sobrino a otro poeta novel, el cual le comunicó a varios aprendices del oficio [...] (MR, 95, RR)

d. [...] entregando su mano, y aún no sé si su corazón, a un honrado mercader de la calle de Postas [...] (MR, 95, RR)

Hasta aquí se ha observado cómo, en textos de autores representativos de los siglos XVIII y XIX, la duplicación clítica tiende a la obligatoriedad con los OOII pronominales, mientras que con otros OOII altamente topicales todavía es mayoritaria la no duplicación; los OOII con un bajo grado de topicidad no se ven en general afectados por este fenómeno por el momento, y quedan al margen del ámbito nuclear de la expansión de la duplicación clítica. Se ha observado también que la duplicación en este período está aún estrechamente ligada a determinados contextos pragmáticos. En adelante se apreciará cómo la duplicación pierde carga pragmática y se hace cada vez más regular.

Así, en Pío Baroja (PB) la duplicación del OI aumenta con respecto a los dos autores anteriores, y supone un $37 \%$ sobre el total de OOII estudiados.

TABLA 5

DCL OI PÍO BAROJA

\begin{tabular}{|l|l|l|l|}
\hline & & DCL & No DCL \\
\hline Animados 57 & & $23(57) \mathbf{4 0} \%$ & $34(57) \mathbf{6 0 \%}$ \\
\hline & Def. Esp. Sg. P. tónico & $7(57) \mathbf{1 2 \%}$ & $1(57) \mathbf{2 \%}$ \\
\hline & Def. Esp. Sg. & $16(57) \mathbf{2 8 \%}$ & $23(57) \mathbf{4 0 \%}$ \\
\hline
\end{tabular}




\begin{tabular}{|l|l|l|l|} 
& Def. Esp. Pl. & --- & $1(57) \mathbf{2 \%}$ \\
\hline & Def. Inesp. Pl. & ---- & $4(57) \mathbf{7 \%}$ \\
\hline & Indef. Esp. Sg. & --- & $2(57) \mathbf{4 \%}$ \\
\hline & Indef. Inesp. Pl. & ---- & $3(57) \mathbf{5 \%}$ \\
\hline Inanimados 7 & & $1(7) \mathbf{1 4 \%}$ & $6 \quad(7) \mathbf{8 6 \%}$ \\
\hline
\end{tabular}

Como se observa en la Tabla 5, la duplicación del OI sigue en Baroja sin alcanzar a los inanimados más que en un ejemplo aislado con un nombre propio:

(15)También le daba mucha importancia a la Curcushada (los cuernos de la luna) $[\ldots](\mathrm{PB}, 87)$

En cuanto a los animados, la duplicación se hace prácticamente obligatoria con los pronombres tónicos salvo en un caso marginal en una construcción pasiva:

(16) Media hora después vino don Matías Cepeda y fui presentado a él. (PB, 119)

La duplicación no avanza más allá de los definidos específicos singulares, y aún entre éstos sigue siendo más frecuente la no duplicación. Un 19\% de las duplicaciones en estos casos se da en relación con complementos afectados (17.a), y un $25 \%$ se da en contextos en los que se necesita aclarar cuáles son los referentes en el discurso (17.b-c). Algunos de estos ejemplos resultan dudosos, como (17.c), y apuntan a que la duplicación comienza a generalizarse sin necesidad de contextos pragmáticamente marcados, o cargados de apreciaciones subjetivas. Así, se aprecia que un $56 \%$ de las duplicaciones de OOII animados definidos específicos no responde a motivación alguna aparente (17.d-f), pues en nada difieren de otros OOII análogos sin duplicación (18. a-c). Este es un paso más hacia la concordancia.

(17)a. [...] como si tuviera miedo de que yo le hiciera algún daño a su padre. (PB, 181)

b. [...] le pregunté yo a Recalde. $(\mathrm{PB}, 96)$

c. - Hace veinte años que no le escribo yo a mi mujer, y seguramente se creerá que me he muerto. $(\mathrm{PB}, 152)$

d. [...] le conté la conversación a mi segundo, e hicimos una serie de indagaciones entre capitanes $[\ldots](\mathrm{PB}, 152)$

e. - Mira, mira - le dije a Recalde. (PB, 109) 
f. El viejo Yurrumendi, un extraño inventor de fantasías, le dijo a Zeyaleta que aquella cueva era un antro donde se guarecía una gran serpiente con alas $[\ldots](\mathrm{PB}, 78)$

(18)a. Varias veces pregunté a Mary si tenía algún proyecto para el porvenir. (PB, 188)

b. Pregunté a mi madre si conocía al retratado, y me dijo que era su hermano Juan [...] (PB, 154)

c. - Da la mano a este caballero. (PB, 186)

Estos datos muestran que en Baroja la duplicación del OOII se extiende con respecto a épocas anteriores, pero no lo hace en relación con la escala de topicidad -no alcanza a OOII con menor grado de topicidad-, sino que permanece en los contextos en los que había comenzado y se libera de cargas pragmáticas dentro de ellos. Según esto, la duplicación del OI está desde un comienzo fuertemente ligada a la topicidad y comienza a avanzar en su gramaticalización como morfema de concordancia dentro de este ámbito, antes de extenderse a otros. De esa manera, y como quedó apuntado al comienzo de esta sección, el clítico se consolidaría primero como "marca pragmática de la concordancia del verbo con el tópico" (Girón Alconchel: 2002: 109) antes de convertirse en un morfema de concordancia del verbo con el OI, lo cual tendrá lugar en todo caso cuando se extienda a todos los OOII sin importar su naturaleza.

Para finalizar este subapartado, en Javier Marías (JM), casi un siglo después, la duplicación del OI prevalece ya sobre la no duplicación, que se produce sólo en el $28 \%$ de los casos. La duplicación continúa fundamentalmente anclada en el mismo ámbito señalado para Baroja: OOII altamente topicales, que representan el $89 \%$ sobre el total de duplicaciones del OI. Los complementos nominales de este tipo duplican sin necesidad de un contexto marcado pragmáticamente en el $93 \%$ de los casos (19), mientras que la duplicación con pronombres tónicos es enfática o contrastiva en un $80 \%(20)$.

(19)a. “¿Qué, cómo va la moqueta atómica?”, le pregunté luego a Ian [...] (JM, 89)

b. [...] le habría preguntado por tanto al señor Stone -a quién si no- si habían recibido algo de Vernon Lee [...] (JM, 139)

c. [...] el novelista Angus Wilson le regaló una de sus novelas al cineasta George Cukor [...] (JM, 110)

(20)a. [...] no habían encontrado mejor explicación que atribuirme a mí-no al narrador sin nombre, sino a Xavier Marías con nombre- la adquisición de 
un extravagante y vicioso hábito de los dons de Oxford del que nunca tuve noticia [...] (JM, 138)

b. Lo mismo que de su vanidad puede decirse de su impertinencia, no se tomará a mal la del otro si éste le consiente a él la suya sin ponerse rígido a las primeras de cambio. (JM, 61)

c. [...] quizá sentía que le tocaba compensar a ella, muchas mujeres se sienten fácilmente en deuda [...] (JM, 132)

Esto ejemplifica lo dicho ya anteriormente: en los complementos pronominales se fija primero la concordancia, de manera que su realización léxica se vuelve superflua. El texto de Marías muestra cómo los complementos nominales siguen a los pronominales de cerca, y concuerdan de manera regular, sin resultar aún del todo redundantes.

Por último, la duplicación ha comenzado a extenderse a los indefinidos específicos (21), y a los inanimados (22), sólo cuando ya ha comenzado a fijarse la concordancia en el ámbito nuclear del fenómeno.

(21)[...] una Universidad norteamericana iba a ofrecerle unos suculentos cursos a un ingrato o poco servil discípulo suyo $[\ldots](\mathrm{JM}, 43)$

(22)[...] se vengaba de una crítica que le hice a su más reciente libro sobre Galdós [...] (JM, 104)

TABLA 6

DCL OI JAVIER MARÍAS

\begin{tabular}{|l|l|l|l|}
\hline & & \multicolumn{1}{|c|}{ DCL } & \multicolumn{1}{|c|}{ No DCL } \\
\hline Animados 37 & & $28(37) \mathbf{7 5 \%}$ & $9(37) \mathbf{2 5 \%}$ \\
\hline & Def. Esp. Sg. P. tónico & $\begin{array}{l}11(37) \mathbf{3 0} \% \\
\text { Enfáticos: } 8(10) 80 \%\end{array}$ & --- \\
\hline & Def. Esp. Sg. & $\begin{array}{l}16(37) \mathbf{4 3} \% \\
\text { No enfáticos: 15 (16) } \\
93 \%\end{array}$ & 8 (37) $\mathbf{2 1} \mathbf{6} \%$ \\
\hline & Indef. Esp. Sg. & $1(37) \mathbf{2 \%}$ & ---- \\
\hline & Plural genérico & ---- & $1(37) \mathbf{3 \%}$ \\
\hline Inanimados 3 & & $1(3) \mathbf{3 3 \%}$ & $2(3) \mathbf{6 7 \%}$ \\
\hline
\end{tabular}

2.2. La extensión de la duplicación clítica en el OD 
En cuanto al OD, cabe decir que Torres de Villarroel aporta un ejemplo de no duplicación ante pronombre tónico. Se trata un contexto negativo, y de un OD plural, lo cual puede explicar la ausencia de duplicación, si bien por otra parte se trata de un contexto contrastivo que podría favorecerla:

(23) [...] no me han oído reñir ni a ellos ni a otro de los familiares [...] (TV, 124)

Un 55\% de los OODD analizados en Mesonero Romanos duplican el clítico, porcentaje parejo al $45 \%$ de no duplicaciones. La duplicación sigue sin ser absolutamente obligatoria con los pronombres tónicos (24.a-b), y está relacionada en un $80 \%$ de los casos con contextos de énfasis pragmático (25.a), con contextos potencialmente ambiguos necesitados de una aclaración (25.b), o con contextos marcados estilísticamente (25.c).

(24)a. [...] son causas bastantes para afirmar a usted en su primera opinión. (MR, 66, EP)

b. [...] que es muy querido mío y de mi pueblo, para servir a Dios y a mí, $\mathrm{y}$ en un tris estuvo el que hubiéramos sido matrimonio [...] (MR, 130, $\mathrm{RV})$

(25)a. [...] y a lo mejor nos asusta por las noches, despertando despavorida y corriendo por toda la casa, diciendo que la persigue la sombra de no sé qué Astolfo o Ingolfo "el exterminador"; y nos llama tiranos a su madre y a mí, y dice que tiene guardado un veneno, no sé bien si para ella o para nosotros $\quad[\ldots](\mathrm{MR}, 91, \mathrm{RR})$

b. No haya miedo el cojuelo Asmodeo, ni su licenciado don Cleofás, que para tal momento solicitemos sus auxilios con el objeto de levantar los tejados de las casas y reconocer lo que pasa en las buhardillas: por la ocasión dejémoslos a los ladrones y enamorados (que también suelen aprovecharse a tales horas de aquel abandono) [...] (MR, 73, PC)

c. [...] y ayudado de mi catalejo, tendí la vista por la dilatada superficie, para ver si la divisaba, no la rápida diligencia, no el brioso alazán, sino la acompasada galera en que debía venir el cuasi escribano [...] (MR, 35, CT)

El ejemplo (25.c) presenta un uso catafórico, propiciado por un estilo poético. Por otra parte, en (25.b) el clítico es necesario para remitir a referentes previamente mencionados -los ladrones-, al tiempo que la aparición del OD léxico permite la inclusión de otros nuevos -los enamorados. 
En Pío Baroja, la duplicación se mantiene obligatoria con los pronombres tónicos sin necesidad de contextos enfáticos. Por otro lado, en Baroja se hace frecuente $(72 \%)$ la duplicación del OD nominal no sólo en casos marcados por el énfasis como (26.a-c), sino también en otros casos que ni aportan una aclaración dentro de un contexto ambiguo, ni parecen verdaderas dislocaciones a la derecha, y que recuerdan más bien a la duplicación generalizada del OD propia del español rioplatense (26.d-f), propiciada seguramente por un valor subjetivo de afectividad:

(26)a. - Pues se le despide a la chica. - No se le puede despedir a la chica. (PB, 166)

b. - Ya le quisiera yo ver al capitán Cook, calvo y con las barbas blancas, venir a esta casa. $(\mathrm{PB}, 145)$

c. Y cuando el barco queda a flote, y todo el mundo dice que es un gran barco, hay que verle a Shempelar haciendo esfuerzos maravillosos [...] (PB, 162)

d. Los chicos le considerábamos a Caracas como un ingeniero naval admirable $[\ldots](\mathrm{PB}, 92)$

e. Y la Cashilda, mientras decía esto, le miraba a Recalde sonriendo, con sus ojos azules. $(\mathrm{PB}, 167)$

f. Después le acompañaría a Don Ciríaco en la derrota de Cádiz a Filipinas $[\ldots](\mathrm{PB}, 114)$

Para concluir, en esta sección se ha analizado la duplicación clítica atendiendo a cómo se produce su extensión: la duplicación se gramaticaliza como afijo de concordancia con los pronombres personales y, en menor medida, con SSNN con un alto grado de topicidad, antes de haber empezado a afectar a complementos inanimados, indefinidos y/o inespecíficos; en otras palabras, el proceso finaliza en un ámbito antes de haber comenzado en otros. Esto nos lleva de nuevo a la idea de que la duplicación es un mecanismo de concordancia con el tópico, independientemente de la función sintáctica. La verdadera conjugación objetiva llegará cuando la duplicación se haya extendido a cualquier tipo de objeto indirecto de manera análoga a como hoy la concordancia con el sujeto se produce incluso cuando éste no es animado, agente o tópico.

\section{EL BAJO GRADO DE TOPICIDAD DEL SUJETO}

En la mayoría de los casos, el contraste entre duplicación y no duplicación puede explicarse atendiendo a las escalas de topicidad y a su interacción con los contextos pragmáticos favorecedores: énfasis, contraste y afectación. No obstante, esto no es siempre suficiente, y entonces se hace necesario atender a la influencia del contexto sintáctico-discursivo para explicar aquellos casos en los que hay ausencia de 
duplicación en contra de lo que sería esperable. El objetivo de esta sección será estudiar cómo las propiedades del sujeto -su mayor o menor grado de topicidadpueden interactuar con la duplicación del objeto. De esta manera, se observará que las construcciones impersonales, así como aquellas con sujetos inanimados o no agentivos, pueden ser entendidas como un factor desfavorecedor de la duplicación. Esta herramienta será empleada para complementar el análisis de ciertos casos problemáticos en las obras de Torres Villarroel y Mesonero Romanos; en ambos autores se abordarán ejemplos de no duplicación en construcciones topicalizadas, y en Mesonero Romanos se analizarán además casos de no duplicación del OI.

\subsection{Ausencia de duplicación con objetos topicalizados en Torres Villarroel.}

Como muestra la Tabla 7, la duplicación clítica tiene una fuerte presencia en Torres Villarroel, pero sólo un 19\% del total de duplicaciones se produce fuera de los contextos de topicalización.

TABLA 7

DCL TORRES VILLARROEL

\begin{tabular}{|l|c|l|l|}
\hline DCL 57(95) 60\% & & No DCL 38 (96) $\mathbf{4 0 \%}$ & \\
\hline Topicalización & $46(57) \mathbf{8 1 \%}$ & Topicalización & $8(38) \mathbf{2 1 \%}$ \\
\hline No topicalización & $11(57) \mathbf{1 9 \%}$ & No topicalización & $30(38) \mathbf{7 9 \%}$ \\
\hline
\end{tabular}

Un 56\% de los ejemplos estudiados son complementos dislocados a la izquierda; de ellos, se duplica el $85 \%$. Puesto que las construcciones topicalizadas son las primeras en duplicar el objeto (directo o indirecto) desde los primeros testimonios medievales, y son el contexto en el que la duplicación resulta obligatoria ya en época moderna, lo llamativo en el corpus de Torres Villarroel es el 15\% de tópicos no duplicados (un $21 \%$ sobre el total de no duplicaciones):

TABLA 8

DCL TOPICALIZACIÓN TORRES VILLARROEL

\begin{tabular}{|l|c|c|}
\hline & $\begin{array}{c}\text { DCL topicalización } \\
46(54) \mathbf{8 5} \%\end{array}$ & $\begin{array}{c}\text { No DCL topicalización } \\
8(54) \mathbf{1 5 \%}\end{array}$ \\
\hline Animado & $26(46) \mathbf{5 7 \%}$ & $2(8) \mathbf{2 5 \%}$ \\
\hline Inanimado & $20(46) \mathbf{4 3} \%$ & $6(8) \mathbf{7 5 \%}$ \\
\hline $\begin{array}{l}\text { Sujetos con bajo grado } \\
\text { de topicidad }\end{array}$ & $2(46) \mathbf{4 \%}$ & $7(8) \mathbf{8 7} \mathbf{5 \%}$ \\
\hline
\end{tabular}


TABLA 9

DCL TÓPICOS ANIMADOS / INANIMADOS TORRES VILLARROEL

\begin{tabular}{|l|c|c|}
\hline & DCL & \multicolumn{1}{|c|}{ No DCL } \\
\hline Total tópicos animados (28) & $26(28) \mathbf{9 3 \%}$ & $2(28) \mathbf{7 \%}$ \\
\hline Total tópicos inanimados (26) & $20(26) \mathbf{7 7 \%}$ & $6(26) \mathbf{2 3 \%}$ \\
\hline
\end{tabular}

Como muestran la tablas 8 y 9, la oposición animado / inanimado es relevante, pero no determinante en este caso: efectivamente, los complementos animados se duplican más que los inanimados, y dejan de duplicarse menos. No obstante, dado que los tópicos inanimados participan más de la duplicación (77\%) que de la no duplicación (23\%) -como se observa en la Tabla 9- la inanimacidad no explica por sí sola el $15 \%$ de tópicos no duplicados en Torres Villarroel.

Entre los casos de no duplicación, un 62'5\% corresponde a sintagmas definidos específicos (entre singulares y plurales), pero ninguno de ellos es además animado (27) -el rasgo más importante en la escala-. Aún así, esos datos no permiten atribuir a la indeterminación o a la indefinitud la causa de la no duplicación. Por otra parte, entre los casos de duplicación un 39\% es indefinido y/o inespecífico (28.a-c); si bien no es un porcentaje muy alto, muestra que la duplicación de este tipo de sintagmas no es en absoluto imposible tratándose de topicalizaciones.

(27) A este dejamiento (que, en mi juicio, es mal humor o filosofia) han llamado soberbia y rusticidad mis enemigos. (TV, 71)

(28)a. Y a los pocos que ruedan los caminos, y a los muchos que trajinan en las ciudades, jamás los temí $[\ldots](\mathrm{TV}, 128)$

b. $[\ldots]$ a los que viven no les pido nada $[\ldots](\mathrm{TV}, 83)$

c. A otras castas de hombres, vigorosos en los vicios o en las virtudes, también les hacen la caridad de inmortalizarlos $[\ldots](\mathrm{TV}, 68)$

No habiendo encontrado hasta aquí una causa verdaderamente determinante en la no duplicación del tópico, es necesario atender a un factor añadido observado en los datos. Como mostraba la Tabla 8 , un $87 ' 5 \%$ de los casos de no duplicación se produce en construcciones impersonales o con un agente indeterminado -sujetos con bajo grado de topicidad-. Si el énfasis pragmático y la afectación del complemento se han señalado como circunstancias favorecedoras de la duplicación, éstas encontrarán difícil acomodo en contextos tales como los de (29). Es más, cabría decir que el grado de topicidad del objeto está en cierta medida determinado por el del sujeto. 
(29)a. A la mayor o menor altura de los afectos y a la más furiosa o sosegada expresión de las pasiones llaman genio $[\ldots](\mathrm{TV}, 121)$

b. Esta descendencia me ha dado Dios $[\ldots](\mathrm{TV}, 85)$

c. $[\ldots]$ a ninguno han ahorcado ni azotado $[\ldots](\mathrm{TV}, 85)$

Sin embargo, como puede observarse, en ningún ejemplo puede decirse que el bajo grado de topicidad del sujeto sea la causa única de la no duplicación. Así, tanto en (29.a) como en (29.b) encontramos complementos inanimados, y (29.c) presenta un contexto negativo de referencia inespecífica. (29.b) tiene, además, un agente expreso realizado como sujeto sintáctico, pero se ha incluido en este grupo de ejemplos por la ausencia de la volición típica de los agentes 9 . No es por tanto posible postular que este factor sea determinante en ningún caso, y tampoco que opere de manera aislada con respecto a los demás.

En cuanto al 4\% de casos de indeterminación del agente entre los ejemplos de duplicación, cabe decir que se trata de casos en los que el autor está hablando en general de un tipo de personas para él despreciable, y convierte en un momento dado a esas personas indeterminadas en el tópico discursivo. Por otra parte, estos ejemplos exhiben el tipo de complemento afectado que puede favorecer la duplicación; (30.b) es además un caso de extrapolación desde una cláusula subordinada, lo cual hace más necesaria aún la duplicación que en el caso de los tópicos normales:

(30)a. [...] y mis cenizas deseo que no me las alboroten [...] (TV, 67)

b. A otras castas de hombres, vigorosos en los vicios o en las virtudes, también les hacen la caridad de inmortalizarlos [...] $(\mathrm{TV}, 68)$

\subsection{Ausencia de duplicación con objetos topicalizados en Mesonero Romanos.}

Mesonero Romanos no tiene en absoluto la predilección por la dislocación a la izquierda que exhibe Torres Villarroel, representando estas construcciones un 19\% de los casos estudiados.

Los complementos topicalizados se duplican en Mesonero Romanos en un 75\% de los casos. El 66\% de los casos de no duplicación corresponde a complementos inanimados, y además plurales (aunque determinados y específicos):

(31)a. A las modestas y filosóficas comedias de Moratín, Gorostiza y Bretón

9 Resultaría anómala una construcción del tipo esta descendencia me la ha dado Dios (a $m i)$, de la que se inferiría una situación en la que Dios haga la entrega en persona. El ejemplo de (29.b) describe una acción proveniente de Dios, pero no efectuada intencionalmente por él. 
habían sustituido en nuestra escena los apasionados dramas [...] (MR, 96, RR, NdelA)

b. [...] a los puestos ambulantes de buñuelos habían sucedido la excitantes pasas $[\ldots](\mathrm{MR}, 46, \mathrm{RSI})$

Sin embargo, también se encuentran ejemplos similares entre los casos de duplicación. Comparando (31.a-b) con (32.a-b) se observa que los ejemplos de (31) presentan agentes inanimados, a diferencia de lo que sucede en (32.a-b). En cambio, en (32.c) se dan exactamente las mismas circunstancias que en (31), y sin embargo se duplica el complemento; en este caso particular, el largo inciso de la relativa, seguido del sujeto y otro inciso, parece ser el factor que determina la duplicación como mecanismo para recuperar el tópico. La distancia entre los elementos oracionales favorece necesariamente la duplicación como concordancia; (32.a) cuenta también con la interpolación de una oración de relativo, y no así los ejemplos de (31).

(32)a. Es fama en el Olimpo que estas horas de reposo, en que el dios de los membrillos cede a su hermana la alta misión de propagar las luces, las tenía consagradas de tiempo inmemorial a tomar las cuentas de cargo y data a las señoras Musas allá en el Parnaso [...] (MR, 98, SP)

b. Las carretas de la Iglesia o del foro no he podido siquiera indicárselas [...] (MR, 67, EP)

c. [...] todas estas manias, que en cualesquiera épocas han debido existir, y sin duda en siglos anteriores habrán podido pasar por extravíos de la razón o debilidades de la humana especie, el siglo actual, más adelantado y perspicuo, las ha calificado de 'romanticismo puro'. (MR, 80, RR)

Por otro lado, aparece además un ejemplo aislado de no duplicación con un sintagma definido, específico y singular (33). En este ejemplo el sujeto sintáctico es inanimado, y no agentivo, pero aún así la no duplicación resulta bastante anómala, y puede en todo caso estar relacionada con el estilo arcaizante que el autor emplea en clave paródica a lo largo de la narración de "El Romanticismo y los Románticos":

(33) [...] usurpando de este modo ora los aplausos, ora los silbidos que a mi sobrino correspondían $[\ldots]$ (MR, 95, RR) 
TABLA 10

DCL TOPICALIZACIÓN MESONERO ROMANOS

\begin{tabular}{|l|c|c|}
\hline & $\begin{array}{c}\text { DCL topicalización } \\
9(12) \mathbf{7 5 \%}\end{array}$ & $\begin{array}{c}\text { No DCL topicalización } \\
3(12) \mathbf{2 5 \%}\end{array}$ \\
\hline Animados & $2(9) \mathbf{2 3 \%}$ & $1(3) \mathbf{3 3 \%}$ \\
\hline Inanimados & $7(9) \mathbf{7 7 \%}$ & $2(3) \mathbf{6 7 \%}$ \\
\hline $\begin{array}{l}\text { Sujeto con bajo } \\
\text { grado de topicidad }\end{array}$ & $1(9) \mathbf{1 1 \%}$ & $3(3) \mathbf{1 0 0 \%}$ \\
\hline
\end{tabular}

TABLA 11

TÓPICOS ANIMADOS / INAINMADOS EN MESONERO ROMANOS

\begin{tabular}{|l|c|c|}
\hline & DCL & No DCL \\
\hline Animados 3(12) & $2(3) \mathbf{6 7 \%}$ & $1(3) \mathbf{3 3 \%}$ \\
\hline Inanimados 9(12) & $7(9) \mathbf{7 8 \%}$ & $2(9) \mathbf{2 2 \%}$ \\
\hline
\end{tabular}

En conclusión, en los siglos XVIII y XIX la topicalización es el contexto dominante en el que se produce la duplicación clítica, pero ésta aún no opera con total obligatoriedad dado que todavía se ve afectada por factores desfavorecedores tales como la inanimacidad o la indefinitud en primera instancia, además del bajo grado de topicidad del sujeto como impedimento añadido.

\subsection{Ausencia de duplicación del OI en Mesonero Romanos}

Como se ha visto hasta aquí, las propiedades del sujeto tienen una influencia significativa, pero no determinante, en la ausencia de duplicación. Un 32\% de los casos de no duplicación de OOII animados en Mesonero Romanos corresponde a ejemplos con agentes inanimados (34.a-c), inespecíficos (34.d) o no volitivos (34.e). En varios casos, esta circunstancia coincide con sintagmas determinados y específicos como el de (34.a).

(34)a. [...] no lejos de él pasa un cabriolé que la bondad marital de un médico dispensó aquella tarde $a$ su esposa $[\ldots](\mathrm{MR}, 55, \mathrm{P})$

b. No hay que decir el contento que todo esto inspiraría a los suyos [...] (MR, 64, EP)

c. La frecuentación de sus calles hará conocer al forastero que todas ellas [...] (MR, 71, PC)

d. ¡Y qué porro debió ser el primero que enseñó a hablar a las cotorras [...]! (MR, 104, SP) 
e. [...] [mi sobrino] llegó muy luego a ser considerado como la estampa más 'romántica' de todo Madrid y a servir de modelo a todos los jóvenes aspirantes $[\ldots](\mathrm{MR}, 82, \mathrm{RR})$

\section{Conclusiones}

En este trabajo se ha realizado un estudio descriptivo del fenómeno de la duplicación clítica en español, atendiendo fundamentalmente a su evolución diacrónica en relación con la noción de 'tópico'. Para ello, se ha empleado un corpus constituído por obras representativas de los siglos XVIII, XIX y XX, dentro del cual se han contrastado los ejemplos de duplicación frente a los de no duplicación. Esto ha permitido analizar de manera precisa los factores favorecedores y desfavorecedores de la duplicación, siempre subordinados a la noción de 'tópicidad'. Los datos ofrecidos en este estudio han servido para ilustrar dos ideas fundamentales:

En primer lugar, se han aportado pruebas en favor de las teorías que consideran la duplicación clítica como un mecanismo de concoradancia con el tópico (Girón: 2002). Así, se ha observado que la duplicación se hace regular en el ámbito de los SSNN con un alto grado de topicidad antes de extenderse a otros ámbitos, es decir, la concordancia no se establece con la función sintáctica de 'objeto' (directo o indirecto) per se, sino que se establece en relación al carácter topical del argumento en cuestión.

En segundo lugar, se ha podido comprobar cómo la incidencia de la noción de tópico sobre el fenómeno de la duplicación clítica rebasa el ámbito del complemento duplicado, y trasciende efectivamente a toda la construcción dentro de la cual se está produciendo el cambio. Es por esto que las propiedades del sujeto -el participante principal de la oración- pueden afectar al comportamiento de un proceso operante en un nivel sintáctico inferior. Así, se ha propuesto en este trabajo que aquellos contextos que presentan un sujeto con un bajo grado de topicidad construcciones impersonales, sujetos inanimados y/o no agentivos- desfavorecen la duplicación clítica del objeto. Si bien se ha podido observar que este factor nunca opera de manera aislada o determinante, ha quedado demostrado que puede ser una herramienta útil para ayudar a explicar ciertos casos en los que la duplicación es esperable y no obstante no se produce.

\section{Obras citadas}

ENRIQUE ARIAS, Andrés: "Spanish object agreement markers and the typology of object agreement morphology", en Steve Dworkin / Dieter Wanner (eds.), New approaches to Old Spanish Issues in Romance Historical Linguistics, Philadelphia, John Benjamins, 2002, pp. 149-164. 
BARRIO DE LA ROSA, Florencio: "Participación y posesión en los dos manuscritos del Libro de Alexandre", en Concepción Company Company y José G. Moreno de Alba (eds.), Actas del VII Congreso Internacional de Historia de la Lengua Española. Vol. 1. Madrid, Arco/Libros, Fundación Banco Santander, Asociación de Historia de la Lengua Española, 2008, pp. 353-367.

BECERRA BASCUÑÁN, Silvia: Estudio diacrónico y sincrónico del objeto indirecto en el español peninsular y de América, Copenhague, Museum Tusculanum Press, University of Copenhague ("Études Romanes 57"), 2006.

FLORES, Marcela / MELIS, Chantal: "OD preposicional y OI duplicado: sobre la vinculación de dos cambios", en Bustos Tovar J. J. / Girón Alconchel, J. L. (eds.), Actas del VI Congreso Internacional de Historia de la Lengua Española, Madrid, Arco Libros, I, 2006, pp. 667-676.

GARCÍA-MIGUEL, José María: "La duplicación del complemento directo e indirecto como concordancia", Verba, 18, 1991, pp. 375-410.

GIRÓN ALCONCHEL, José Luis: "Procesos de gramaticalización del español clásico al moderno", en M ${ }^{\mathrm{a}} \mathrm{T}$. Echenique Elizondo y J. Sánchez Méndez (eds.), Actas del $V$ Congreso Internacional de Historia de la Lengua Española, I, Madrid, Gredos y CAM (Caja de Ahorros del Mediterráneo), 2002, pp. 103-121.

GIVÓN, Talmy: "Topic, pronoun and grammatical agreement", en Charles Li (ed.), Subject and topic, Nueva York, Academic Press, 1976, pp. 149-188.

IBÁNEZ CERDÁ, Sergio: "El papel del clítico 'le' en las construcciones de duplicación de dativo. Evidencia diacrónica", en Concepción Company Company y José G. Moreno de Alba (eds.), Actas del VII Congreso Internacional de Historia de la Lengua Española. Vol. 1. Madrid, Arco/Libros, Fundación Banco Santander, Asociación de Historia de la Lengua Española, 2008, pp. 719-730.

LLORENTE, Antonio y José MONDÉJAR: "La conjugación objetiva en español”, RESEL, 4/1, 1974, pp. 1-60.

SUÑER, Margarita: "The role of agreement in clitic doubled constructions", Natural Language and Linguistic Theory, 6, 1988, pp. 391-434.

TESNIÈRE, Lucien (1959): Éléments de syntaxe structurale, Klincksieck, Paris, 1969.

WEISSENRIEDER, Maureen: "Indirect object doubling: saying things twice in Spanish”, Hispania, 78, 1995, pp. 169-177.

\section{CORPUS}

BAROJA, Pío. Las inquietudes de Shanti Andía. Edición de Julio Caro Baroja. Madrid: Cátedra, 1989.

MARÍAS, Javier. Negra espalda del tiempo. Madrid: Círculo de Lectores, 1998. 
MESONERO ROMANOS, Ramón de: Escenas Matritenses, Edición de E. Correa Calderón. Madrid: Anaya, 1970.

TORRES VILLARROEL, Diego de. Vida. Edición de Manuel María Pérez López. Madrid: Espasa Calpe, 1989. 